incarceration, scrotal edema, hematoma spermatic cord and paralytic ileus.

\section{CRYPTORCHIDISM AND THE VALUE OF ULTRASOUND SCAN}

doi:10.1136/archdischild-2012-302724.1600

M Shoukry, K Pojak, M Haddad, M Choudhry. Paediatric Surgery, Chelsea and Westminster Hospital, Imperial College, London, UK

Aim To evaluate the usefulness and accuracy of Ultrasound Scan (USS) in investigating cryptorchidism.

Methods Review of all children with cryporchidism who had undergone USS examination for their testes from February 2010 to October 2010. Preoperative USS findings for presence or absence and position of testes were noted and compared to the operative findings. Sensitivity and specificity of USS examination were calculated.

Results 50 testes in 42 boys were examined by USS preoperatively during the study period. Median age was 6 years. All had single B-mode USS examination using Grey scale and Doppler images. 37 out of 50 testes were palpable on examination under anaesthesia and underwent single stage orchidopexy. All impalpable testes had diagnostic laparoscopy. 2 out of 13 were found atrophic hence excised and rest had staged orchidopexy. USS and operative findings are summarised in the table attached.

The sensitivity and specificity of USS in determining the presence of testes were $88 \%$ and $4 \%$ respectively. Positive predictive value was $52 \%$ and negative predictive value was $25 \%$. The sensitivity and specificity of USS in localisation of testicular position were $77 \%$ and $4 \%$ respectively. Positive predictive value was $32 \%$ and negative predictive value was $16 \%$.

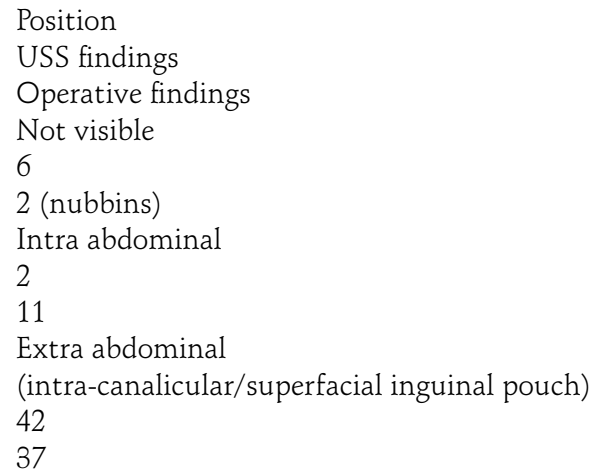

Conclusion USS Has Low Sensitivity and Specificity in Determining the Presence of Testes and Localisation of Its Position. In the Presence of Examination Under Anaesthesia and Laparoscopic Technique, USS is Not Recommended in Cyptorchidism.

\section{TYMPANOSTOMY AND ADENOIDECTOMY FOR TREATMENT OF OTITIS MEDIA IN CHILDREN}

doi:10.1136/archdischild-2012-302724.1601

'S Diacova, ${ }^{2} \mathrm{~V}$ Desvignes, 'A Chiaburu, 'D Chirtoca, 'S Parii. 'Otorhinolaryngology, Pediatric Clinic, SMPhU, Chisinau, Moldova; 'Society 'Médecins du Monde', ClermontFerrand, France

Background and Aims Tympanostomy and adenoidectomy are the most frequently performed surgical procedures in children, which are indicated in otitis media with effusion (OME) and recurrent otitis media ( $R O M)$.

The Aim of our research is to analyze the effectiveness, complications and outcome of tympanostomy and adenoidectomy in treatment of otitis media.

Subjects and methods Children at age 1-7 years of life with chronic OME and ROM with the disease duration 6 months and more and complete clinical, audiological and otoscopical evaluation received medical treatment according to our scheme. The absence of positive results after the treatment was the condition for including in the Project 687 children (506 with OME and 181 with ROM). Tympanostomy was performed according to elaborated principles concomitant with adenoidectomy. In 12-18 months we removed tubes and revised the tympanic cavity.

Audiological, clinical and otoscopical assessment was carried out before and every three months during 2 years after surgery.

Results Before surgery the mean hearing level was $33 \mathrm{~dB}$ (SD 3.1 $\mathrm{dB}$ ), after surgery - $13 \mathrm{~dB}$ (SD 2.7) in $93 \%$ of cases during all period of follow up. In $3 \%$ of ears we noted aggravation of ROM in 3 months after surgery, in $2 \%$ - in 6-9 months and in 2\% - in 10-15 months after surgery. Revision of tympanic cavity showed disappearing of chronic changes in $95 \%$.

Conclusions Complex surgical treatment including tympanostomy according to elaborated principles concomitant with adenoidectomy is an effective method of otitis media treatment in children.

\section{ABDOMINAL CYSTIC LYMPHANGIOMAS: RARE LESIONS WITH A VARIABLE PRESENTATION. REPORT OF 3 CASES}

doi:10.1136/archdischild-2012-302724.1602

ML Conighi, ME Michelini, A Franchella. Pediatric Surgery, Universitary Hospital S. Anna, Ferrara, Italy

Background and Aims Abdominal cystic lymphangiomas are uncommon congenital benign tumors. $90 \%$ are diagnosed by the age of 2 years. Retroperitoneal and mesenteric localizations account for less than $5 \%$ of paediatric lymphangiomas. Clinical presentation is variable: they can be incidental findings in prenatal or postnatal period, they can be symptomatic and present with chronic nonspecific abdominal signs (pain, progressive distension), but most commonly they present acutely (infection, haemorrhage, rupture, torsion). We retrospectively reviewed three cases.

Materials and Methods Patient 1 had a prenatal diagnosis of intraperitoneal cystic mass; he underwent postnatal ultrasound follow up and RMN evaluation; at the age of 5 month was performed a laparoscopic exploration, in another hospital, but no mass was identify. We conducted a videoassisted resection of a mesenteric cystic lymphangiomas at the age of 7 months. Patient 2 (3 years and 8 months) and patient 3 (5 years and 10 months) presented with acute abdominal sign; laboratory tests and radiological and ultrasound evaluations were performed. Both patients underwent emergent laparotomy that permitted excision of a giant retroperitoneal cystic lymphangioma.

Results Postoperative recovery was uneventful. Follow up consisted on clinical and ultrasound monitoring. Patients have no recurrence.

Conclusions Complete surgical excision represents the treatment of choice for abdominal cystic lymphangiomas. Prognosis is excellent after surgery; a low recurrence rate exists when there is not a radical resection.

\section{ANGIOGRAPHIC EMBOLIZATION AND TRAUMA: SUCCESSFUL TREATMENT IN TWO ADOLESCENTS}

doi:10.1136/archdischild-2012-302724.1603

${ }^{1} \mathrm{ML}$ Conighi, ${ }^{2} \mathrm{R}$ Galeotti, ${ }^{1} \mathrm{~A}$ Franchella. ${ }^{1}$ Pediatric Surgery; ${ }^{2}$ Interventional Radiology, Universitary Hospital S. Anna, Ferrara, Italy

Background and Aims Since 1990s angiographic embolization is used in adult trauma for bleeding control and organ preservation. Experience in children is limited probably because of a higher success rate of non operative management for solid organ injuries (96-100\%) and for technical limits due to smaller size of arteries. We 